\title{
Fractal growth of tumors and other cellular populations: linking the mechanistic to the phenomenological modeling and vice versa*
}

\author{
Alberto d'Onofrio ${ }^{1}$ \\ July 72006 \\ ${ }^{1}$ Division of Epidemiology and Biostatistics, European Institute of Oncology, Via Ripamonti 435, \\ Milano, Italy, I-20141 \\ E-mail: donofrio@mail.dm.unipi.it, Phone:+390257489819
}

\begin{abstract}
In this paper we study and extend the mechanistic mean field theory of growth of cellular populations proposed by Mombach et al in [Mombach J. C. M. et al., Europhysics Letter, 59 (2002) 923] ( $M L B I$ model), and we demonstrate that the original model and our generalizations lead to inferences of biological interest. In the first part of this paper, we show that the model in study is widely general since it admits, as particular cases, the main phenomenological models of cellular growth. In the second part of this work, we generalize the $M L B I$ model to a wider family of models by allowing the cells to have a generic unspecified biologically plausible interaction. Then, we derive a relationship between this generic microscopic interaction function and the growth rate of the corresponding macroscopic model. Finally, we propose to use this relationship in order to help the investigation of the biological plausibility of phenomenological models of cancer growth.
\end{abstract}

Keywords: Cell - Population - Tumor - Fractal - Mathematical Models

\section{Introduction}

Human beings, animal and plants are sets of cells. As a consequence, modeling the growth of cellular populations should be considered among the most important scientific topics. Surprisingly, the vast majority of theoretical work on this subject [1] produced essentially mathematical models based on qualitative "macroscopic" biological reasoning or, as in the case of the well known Gompertz model, purely on the ground of good fit to experimental data [1. Among the few works aimed at introducing a mechanistic theory in order to link macroscopic to microscopic parameters, the model (hereafter referred as the MLBI model) proposed by Mombach et al. 2] is particularly interesting because of its simplicity and biological plausibility, being based on the realistic hypothesis of long range interactions between cells in a population whose "structure is a fractal" [2]. Furthermore, relying on the fractal structure of cells aggregates, is adequate to describe tumor growth 3, 4, at least in some particularly relevant biological frameworks. As a consequence of its biological realism, MLBI model allowed its authors to unify at microscopic level three well known growth laws (logistic, gompertzian and exponential laws [5]). In other words, apparently contradictory growth models are simply macroscopic different manifestations of a common physical microscopic framework (note that at macroscopic level it is easy to show that those models are linked). However, many other relevant growth laws were

\footnotetext{
*I wish to dedicate this work to my friends and coworkers Prof. Alessandro Bertuzzi and Prof. Alberto Gandolfi, who are among the best people I know, and not only in the scientific world. Of course, they also helped me in revising the draft of this manuscript, and I thank them very much.
} 
proposed. Among them we cite the von Bertanlaffy's [5], the West-Guiot law [6, 8, the power law [9, 10, 11, all of which well describe experimental "in vitro" and "in vivo" data [5, 9, 11].

This "proliferation" of different models, all, at least apparently, theoretically sound, and with some encouraging experimental evidences, should not surprise the non biologist reader. For example, let us consider solid tumors (to which we shall mainly refer in the following): with the term "solid tumors" one summarizes a wide range of polymorphic diseases characterized by at least three levels of growth behaviors: 1)disease-specific level: different kinds of tumors may exhibit different kinds of growth; 2)the inter-patients level: two similar subjects, suffering of the same kind of cancer, may show very different time courses of the diseases; 3)intra-patient level: the main tumor may have growth characteristics that are different from those of its metastases. Finally, there is also a temporal level: tumor is a dynamic disease whose characteristics of proliferation may change reflecting changes in its topological structure [8].

The starting point of this research has been the following question: given the complexity of growth phenomena of tumor and non-tumor cell populations, are the encouraging results obtained by many and different mathematical models simply due to their fitting flexibility or there is some unifying mechanism?

Aim of this letter is to show that the $M L B I$ model is able to unify also the above listed important models of growth, and others, besides those considered in 2. Thus, we shall show that apparently very different behaviors in very similar conditions simply reflect slight differences in physically meaningful (and, in principle, experimentally measurable) parameters such as the fractal dimension of the tumor. Analyzing the relationships between the $M L B I$ model and the model 8 ] we shall also shortly examine the case of time varying topological properties.

The relevance of our findings, in our opinion is twofold. In fact, on one hand, the fact that the $M L B I$ model may particularize itself to all main empirical models, confirmed by good fitting of experimental data, gives more biological soundness to the $M L B I$ model; on the other hand, those models have now a theoretical ground which might explain them at microscopic level.

Furthermore, we shall extend the $M L B I$ model by allowing very general laws of cell-cell interaction and, in so doing, transforming it in a meta-model, that unifies many other models, and, starting from the micro level, to define new ones. We propose a simple method of "reverse engineering", which might allow insights on the biological basis of a given phenomenological model of growth of cellular populations. In fact, we shall show that our generalization of the $M L B I$ model seems to indicate that, for a macroscopically defined model, its population level growth rate (PLGR) must be not only decreasing with the population size, as it is well known, but it must also be convex (its second derivative must be positive).

\section{Phenomenological models and the $M L B I$ model}

A phenomenological model that describes the growth of a population of cells may be written as:

$$
x^{\prime}=x R(x)
$$

where $x$ is the size of the population. $R(x)$ denotes the $P L G R$, which, due to evident biological reasons, must be a decreasing function of $x$.

Coming to the $M L B I$ theory, the assumptions on which the model is built (other than the above mentioned long range cell-cell interactions and the fractal spatial structure of the population) are the following: i)a sufficiently high supply of nutrients (as a consequence the $M L B I$ model is particularly adequate to describe the behavior of an "in vitro" sufficiently nourished multicellular spheroid and also "in vivo" tumors in the angiogenic phase); ii)the inhibiting chemicals are such that they diffuse in the cellular structure; iii)the individual "cell level" proliferation rate of a cell results from its baseline replication rate $G_{i}$ minus the long range inhibition interactions with all other cells of the population. For the generic "i-th" cell it is [2]:

$$
R_{i}=G_{i}-\sum_{j=1}^{n} J \frac{1-\delta_{i, j}}{\left|\overrightarrow{r_{i}}-\overrightarrow{r_{j}}\right|^{\gamma}}
$$


where: $J$ is a constant related to the effectiveness of the inhibitory action, $\left|\overrightarrow{r_{i}}-\overrightarrow{r_{j}}\right|$ is the distance between cell $i$ and cell $j, \delta_{i, j}$ is the Kroeneker's symbol. The function $J\left|\overrightarrow{r_{i}}-\overrightarrow{r_{j}}\right|^{-\gamma}$ is an ansatz function, physically motivated in [2] by the theory of diffusion in fractal structures [12. Starting from (2) and from the assumption of fractal structure (with fractal dimension $D_{f}$ ), in [2] the following relation has been obtained:

$$
R(x)=\bar{G}-J I(x)
$$

where $\bar{G}$ is the average of the $G_{i}$ s and:

$$
I(x)=\frac{\omega}{D_{f}-\gamma}\left(\left(\frac{D_{f}}{\omega} x\right)^{1-\gamma / D_{f}}-1\right) \text { if } \gamma \neq D_{f}, I(x)=\frac{\omega}{D_{f}} \log \left(\frac{D_{f}}{\omega} x\right) \text { if } \gamma=D_{f},
$$

where the constant $\omega$, defined in 2, is related to the density of the cellular aggregate. Thus, starting from microscopic cell-cell interactions, a macroscopic but "non phenomenological" equation $x^{\prime}=$ $x R(x)$ has been obtained. Note that, as previously stressed in [2], the parameters $J$ and $\gamma$ might be determined by concentration measurements, and $D_{f}$ by the pattern of the cellular structure.

The family of ODE models $x^{\prime}=x R(x)$ with PLGR given by (3) and (4) has been studied in [2] with the following results: for $\gamma=D_{f}$ the model reduces to the Gompertz model; for $0 \geq \gamma<D_{f}$ the $M L B I$ model gives the generalized logistic model $x^{\prime}=k_{1} x-k_{2} x^{\nu}$ with $\nu=2-\left(\gamma / D_{f}\right) \leq 2$. However, $\nu=2 \Rightarrow \gamma=0$ i.e. biologically unrealistic constant interactions [2. We add here that this non-realism might explain the poor performances of the logistic model in fitting experimental tumor data, as reported in [5].

\section{The general behavior for $\gamma>D_{f}$}

Unfortunately, because of a minor error in 2, the exploration of the parameter space was not deepened as it deserved. In fact, the authors claimed that for $\gamma>D_{f}$ the behavior of the system is of exponential type. We detected the trivial error and discovered that all the other main phenomenological models of growth may be considered as particular cases of the $M L B I$ model.

If $\gamma>D_{f}$, let us set $\gamma=D_{f}(1+Q)$ with $Q>0$, that yields:

$$
R(x)=\frac{J}{Q}\left(\frac{\omega}{D_{f}}\right)^{Q+1} x^{-Q}+\bar{G}-\frac{J \omega}{D_{f} Q} .
$$

Note that the above $P L G R$ is decreasing and also convex:

$$
R^{\prime}(x)=-J\left(\frac{\omega}{D_{f}}\right)^{Q+1} x^{-Q-1}<0, R^{\prime \prime}(x)=(Q+1) J\left(\frac{\omega}{D_{f}}\right)^{Q+1} x^{-Q-2}>0
$$

We shall show in the final section that the convexity is as important as the negativity of the derivative $R^{\prime}(x)$.

The solution of $x^{\prime}=x R(x)$ when :

$$
\bar{G}-\frac{J \omega}{D_{f} Q}<0 \Rightarrow D_{f}<\gamma<D_{f}+\frac{\omega J}{\bar{G}}
$$

is not the exponentially increasing one given in [2], since the equation $x^{\prime}=x R(x)$ has a global attractor:

$$
\lim _{t \rightarrow+\infty} x(t ; x(0))=x_{e q}=\frac{\omega}{D_{f}}\left(\frac{J \omega}{J \omega-\bar{G} Q D_{f}}\right)^{1 / Q}=\frac{\omega}{D_{f}}\left(\frac{J \omega}{J \omega-\bar{G} \gamma+\bar{G} D_{f}}\right)^{D_{f} /\left(\gamma-D_{f}\right)}
$$

and the dynamics of $x(t)$ is as follows:

$$
x(t)=\left(x_{e q}^{Q}+\left(x(0)^{Q}-x_{e q}^{Q}\right) \operatorname{Exp}\left(-t *\left(-\bar{G} Q+\frac{J \omega}{D_{f}}\right)\right)\right)^{1 / Q}
$$


On the contrary, if $\gamma>\gamma^{*}:=D_{f}+\omega J / \bar{G}$ there is exponential explosion of the growth:

$$
x(t) \propto \operatorname{Exp}\left(\left(\bar{G}-\frac{J \omega}{\gamma-D_{f}}\right) t\right)
$$

In view of some experimental findings, exponential growth has been traditionally associated to initial stages of growth. Thus eq. (10) may be read as follows: the case $\gamma>\gamma^{*}$ is not likely to be observed or it is related to very quickly growing tumors for which a long temporal observation is, of course, not possible.

\section{Power law growth}

Recently, by means of a phenomenological approach and without explicitly assuming the presence of a necrotic core, Hart et al 9] formulated an interesting model whose solution is a power law growth of the tumor mass, and validated this model by means of fitting to breast cancer data. A similar behavior was also predicted in [10, where an interesting individual based model was used, and in [11, where also experimental data are reported (see also [1]). So an important feature for a unified mechanistic model would be to allow such a behavior. Actually, this happens in our case, since if:

$$
\bar{G}-\frac{J \omega}{D_{f} Q}=0 \Rightarrow \gamma=D_{f}+\frac{J \omega}{\bar{G}}
$$

the tumor will grow following an asymptotically power law. In fact, when (11) holds:

$$
x^{\prime}=\frac{J}{Q}\left(\frac{\omega}{D_{f}}\right)^{Q+1} x^{1-Q} \Rightarrow x(t)=\left(x_{o}^{J \omega /\left(D_{f} \bar{G}\right)}+J\left(\frac{\omega}{D_{f}}\right)^{1+J \omega /\left(D_{f} \bar{G}\right)} t\right)^{D_{f} \bar{G} /(J \omega)} .
$$

For large times:

$$
x(t) \propto t^{\frac{\bar{G}}{J \omega}} D_{f}
$$

Note that the power depends on the fractal features of the aggregate of growing cells. In particular, linear growth of the diameter of the spheroid, corresponding to cubic variation of the cell number (quadratic in 2D), is obtained when $\gamma=4 D_{f} / 3$ (in 2D: $\gamma=3 D_{f} / 2$ ). According to [9], the power law growth we have found does not depend on the localization of the proliferating cells near the surface of the spheroid. We remark that our analysis predicts the possibility of power law growth only for a specific combination of the parameters. This might be read in a dichotomic way: either the model has to be modified to manage the power law more robustly, or the power law may be read as a limit case. The same considerations hold for the Gompertz model.

Summarizing the findings of this and of the previous sections, we may state that $\gamma$ is a bifurcation parameter with threshold value $\gamma^{*}=D_{f}+J \omega / \bar{G}>D_{f}$ determining a "catastrophic" transition, since $x(t)$ is bounded for $\gamma<\gamma^{*}$, whereas the growth is unbounded for $\gamma \geq \gamma^{*}$. The threshold for the unbounded growth depends not only on the geometrical parameter $D_{f}$, as stated in [2] (where the threshold was $D_{f}$ ), but also on the parameter $J$ (with $\partial \gamma^{*} / \partial J>0$ ) and on the proliferation parameter $\bar{G}$ (with $\partial \gamma^{*} / \partial \bar{G}<0$ ). Thus, higher values of the proliferation parameter imply a lower threshold for the spatial decaying $\gamma$, whereas high values of $J$ require higher values for $\gamma$ to have power law or exponential expansion.

\section{$5 \quad$ Linear growth law and linear growth}

For $Q=1$, i.e. $\gamma=2 D_{f}$, the law of the growth $x^{\prime}=x R(x)$ becomes a linear first order equation:

$$
x^{\prime}=J\left(\frac{\omega}{D_{f}}\right)^{2}+\left(\bar{G}-\frac{J \omega}{D_{f}}\right) x .
$$


To the best of our knowledge, this is the first time that the possibility of such a simple law of growth is contemplated in the context of tumor growth and with a plausible mild constraint on parameters. If $\bar{G}-J \omega / D_{f}<0$ the tumor size will be attracted to the equilibrium point:

$$
x_{e q}=\frac{J \omega^{2}}{D_{f}\left(J \omega-\bar{G} D_{f}\right)}
$$

or, if $\bar{G}-J \omega / D_{f}>0$, it will expand exponentially. If $\bar{G}-J \omega / D_{f}=0$, the growth of the tumor is linear from $t=0$ :

$$
x(t)=x(0)+J\left(\frac{\omega}{D_{f}}\right)^{2} t .
$$

\section{Comparison with the model by del Santo, Guiot et al. [6, 8]}

We show here that the very interesting model of growth proposed by del Santo, Guiot and coworkers in 6, 8, is a particular case of the $M L B I$ model. In fact the model in [6] reads as follows:

$$
x^{\prime}=x\left(a x^{p-1}-b\right)
$$

with $p \in(2 / 3,1), a>0$ and $b>0$. Matching with (5);

$$
p=2-\frac{\gamma}{D_{f}}
$$

which leads to :

$$
1<\frac{\gamma}{D_{f}}<\frac{4}{3}, \frac{\omega J}{\bar{G}}>\frac{D_{f}}{3}
$$

For tridimensional tumors following the Guiot's law this implies that it must be $\gamma<4$.

Up to now we assumed that the topological properties of the tumor were constant. On the contrary, as Guiot et al stressed in their paper, these properties, and namely the fractal dimension, change during tumor development, in a way that the scaling exponent $p$ is increasing at least for well perfused tumors in the angiogenic phase (i.e. when there is no lack of nutrients, as required for the rigorous application of the $M L B I$ model). This means to assume an increasing $D_{f}(t)$ or, if we assume that also $\gamma$ is a time function, to assume that $D_{f}(t)$ increases faster than $\gamma(t)$. Finally, tumor for which data fitting shows constant $p$ should have $\gamma(t) \propto D_{f}(t)$.

\section{The von Bertanlaffy's model}

One among the early mathematical models of growth, due to von Bertanlaffy [ $[\mathbf{5}$, was as follows:

$$
x^{\prime}=x\left(a x^{-1 / 3}-b\right)
$$

and it is easily recovered as a particular case of the $M L B I$ model provided that:

$$
\gamma=\frac{4}{3} D_{f}, \frac{\omega J}{\bar{G}}>\frac{D_{f}}{3}
$$

\section{Relationships between $\gamma$ and $D_{f}$}

We saw that for $\gamma=D_{f}+(J \omega) / \bar{G}$ there is power law growth, and for higher values of the fractal dimension the growth is more rapid, which well agrees with usual tumor behavior where a high $D_{f}$ is considered an index of high growth velocity (and, as a consequence, of poor survival) [3, 13]. However, for $D_{f}<\gamma<D_{f}+(J \omega) / \bar{G}$, if we use eq. (8) we obtain that: $\partial_{D_{f}} x_{e q}\left(D_{f}, \gamma\right)<0$, and when $\gamma>D_{f}+(J \omega) / \bar{G}$ it is $\partial_{D_{f}} x\left(t ; D_{f}, \gamma\right)<0$. Apparently, the model seems to fail in reproducing an important biological property. However, we note that in the Guiot's and in the von Bertanlaffy's case, 
where there is a linear relationship between $\gamma$ and $D_{f}$, it is $\left(d / d D_{f}\right) x_{e q}\left(D_{f}, \gamma\left(D_{f}\right)\right)>0$. Similarly, for $\gamma>\gamma^{*}$, one may find linear relationships such that the exponential grows faster. Finally, for for $\gamma<D_{f}$ for whatever $\gamma=h\left(D_{f}\right)$ with $h^{\prime}\left(D_{f}\right)>0$ it is: $\left(d / d D_{f}\right) x_{e q}\left(D_{f}, h\left(D_{f}\right)\right)<0$. Summarizing these findings, it seems that it should be $\gamma>D_{f}$ and that $\gamma$ and $D_{f}$ must be positively correlated.

\section{Extending the $M L B I$ model}

We may observe that the inhibition function $\left|\overrightarrow{r_{i}}-\overrightarrow{r_{j}}\right|^{-\gamma}$ used in (2), may assume very high values in the neighboring of the $i$ cell. Furthermore, that particular ansatz was chosen in [2] because of the assumption of diffusion of inhibiting chemicals, which might be no more valid in case of well vascularized tumors. Thus, we change the function $J\left|\overrightarrow{r_{i}}-\overrightarrow{r_{j}}\right|^{-\gamma}$ with another generic positive and decreasing decreasing ansatz function $F\left(\left|\overrightarrow{r_{i}}-\overrightarrow{r_{j}}\right|\right)\left(F(u)>0, F^{\prime}(u)<0, F(+\infty)=0\right.$ and, if it is necessary, $F(0)<+\infty)$. One has:

$$
R_{i}=G_{i}-\sum_{j=1}^{n}\left(1-\delta_{i, j}\right) F\left(\left|\overrightarrow{r_{i}}-\overrightarrow{r_{j}}\right|\right)
$$

Since the spheroid radius is related to $x$ by the following formula (ref. [2], pag. 925): $R_{\max }=$ $\left(x D_{f} / \omega\right)^{1 / D_{f}}$, and proceeding as in [2] but using $F(r)$ instead of the specific ansatz $J r^{-\gamma}$, we easily obtain a generalized $P L G R, R_{g e n}$ :

$$
R_{g e n}(x)=\bar{G}-\omega \int_{r o}^{R_{\max }(x)} F(r) r^{D_{f}-1} d r
$$

which defines a very general family of models of growth. This family is such that all the models belonging to it share two important properties: $R_{g e n}(x)$ is decreasing and convex:

$$
R_{g e n}^{\prime}(x)=-F\left(\left(\frac{D_{f}}{\omega} x\right)^{1 / D_{f}}\right)<0 ; R_{g e n}^{\prime \prime}(x)=-\frac{1}{D_{f}}\left(\frac{D_{f}}{\omega}\right)^{1 / D_{f}} x^{-1+1 / D_{f}} F^{\prime}\left(\left(\frac{D_{f}}{\omega} x\right)^{1 / D_{f}}\right)>0
$$

Note that:

$$
\operatorname{Signum}\left(R_{\text {gen }}^{\prime \prime}(x)\right) \Leftrightarrow-\operatorname{Signum}\left(F^{\prime}\left(x^{1 / D_{f}}\right)\right)
$$

We think that the relationship (25) has implications of relevant biological interest. In fact, as long as the basic hypotheses stated in $[2$ are valid, the $\Leftarrow$ relationship means that the assumption of bio-physically realistic interaction between the cells (i.e. interactions decreasing with the distance) implies that the PLGR of the corresponding mean field macroscopic growth model is convex. On the contrary, a concave $P L G R$ would correspond to a unrealistic $F$. The $\Rightarrow$ relationship may be read as follows: given a macroscopic growth model, if its $P L G R$ is convex, then the underlying microscopic cell-cell interaction is decreasing, which is biologically meaningful. On the contrary, if $R^{\prime \prime}(x)<0$ then it would correspond to an unrealistic cell-cell interaction increasing as the distance between the cells increases.

More explicitly, we propose the study of the convexity of $R(x)$ (i.e. the sign of $R^{\prime \prime}(x)$ )as a way to start investigating the biological plausibility of a phenomenological cellular growth model, and to classify a model characterized by negative or varying sign $R^{\prime \prime}(x)$ at least as "suspect". An example is the well known generalized logistic model with exponent $\nu>2: x^{\prime}=a x-b x^{\nu}$. This model has been introduced in 14, in a context (populations of insects) where values $\nu \geq 2$ are perfectly biologically plausible. Analyzing its $P L G R R(x)=a-b x^{\nu-1}$, it is easy to see that this kind of logistic model would correspond to a non-realistically increasing cell interaction function: $\nu>2 \Rightarrow \gamma<0 \Rightarrow F^{\prime}(r)=$ $(-\gamma) r^{-\gamma-1}>0$. A different example is the following: let us consider an exponentially decreasing PLGR: $R(x)=A \operatorname{Exp}\left(-B x^{d}\right)-C, 0<d \leq 1$. This $P L G R$ is decreasing $\left(R^{\prime}(x)<0\right)$ and convex $\left(R^{\prime \prime}(x)>0\right)$, as it is easy to verify. Furthermore, it would correspond to a decreasing interaction function $F(r)=r^{-D_{f}(1-d)} \operatorname{Exp}\left(-q r^{d * D_{f}}\right)$, which may be read as the $r^{-\gamma}$ function damped by a decreasing exponential. For $d=1$ we have the particular case $F(r)=\operatorname{Exp}\left(-q r^{D_{f}}\right)$. Finally, let 
us consider the Wheldon model [15] in which $R(x)=-w+a /(1+b x)$, which, not surprisingly, is found to be convex $\left(R^{\prime \prime}(x)>0\right)$ and which, as it is easy to verify, is related to the inhibition function $F(r)=1 /\left(1+\hat{b} r^{D_{f}}\right)^{2}$.

\section{Concluding remarks}

Our extension of [2], aimed to stress the role of the cell-cell interactions, neglects important phenomena and may be further extended to model them. For example, the interaction with the microenvironment may be encoded in the parameters $\bar{G}$ and $J$, either as noisy fluctuations or as deterministic dependence on new state variables (e.g. the density of blood microvessels). Some extensions might be less trivial, e.g. introducing subpopulations of quiescent and necrotic cells. This extension would be important in order to relax the basic hypothesis [2] of a sufficiently high level of nutrients, and to include the Gompertz law and the power law growth in a more robust way.

\section{References}

[1] Araujo RP, McElwain DLS. A history of the study of solid tumour growth: The contribution of mathematical modelling. Bulletin of Mathematical Biology 2004;66:1039-1091.

[2] Mombach JCM, Lemke N, Bodmann BEJ, Idiart MAP. A mean-field theory of cellular growth. Europhysics Letters 2002;59:923-928.

[3] Norton L. Conceptual and practical implications of breast tissue geometry: toward a more effective, less toxic therapy. The Oncologist 2005;10:370-381.

[4] Waliszewski P, Konarski J. The Gompertzian curve reveals fractal properties of tumor growth. Chaos, Solitons \& Fractals 2003; 16:665-674.

[5] Marusic M, Bajzer Z, Freyer JP, Vuk-Pavlovic S. Analysis of growth of multicellular tumor spheroids by mathematical models. Cell Proliferation 1994; 27:73-94.

[6] Guiot C, Degiorgis PG, Delsanto PP,Gabriele P, Deisboeck TS. Does tumor growth follow a "universal law"? Journal of Theoretical Biology 2003;225:147-151.

[7] Waliszewski P, Konarski J, Molski M. On the modification of fractal self-space during cell differentiation or tumor progression. Fractals 2000;8(2):195203.

[8] Guiot C, Delsanto PP, Carpinteri A, Pugno N, Mansury Y, Deisboeck TS. The dynamic evolution of the power exponent in a universal growth model of tumors. ArXivq-bio 2005; Paper n. arxivq-bio.QM/0502001

[9] Hart D, Shochat E, Agur Z. The growth law of primary breast cancer as inferred from mammography screening trials data. British Journal of Cancancer 1999;78:382-387.

[10] Drasdo D, Hoehme S. Individual-based approaches to birth and death in avascular tumors. Mathematical and Computer Modelling 2003; 37:1163-1175.

[11] Bru A, Albertos S, Subiza JL, Garcia-Asenjo JL, Bru I. The universal dynamics of tumor growth. Biophysics Journal 2003;85:2948-2961.

[12] O'Shaughnessy B, Procaccia I. Analytical solutions for diffusion on fractal objects. Physical Review Letters 1985; 54:455-458.

[13] Delides A, Panayiotides I, Alegakis A, Kyroudi A, Banis C, Pavlaki A, Helidonis E, Kittas C. Fractal dimension as a prognostic factor for laryngeal carcinoma. Anticancer Research 2005; 25:2141-2144. 
[14] Gilpin ME, Ayala FJ. Global models of growth and competition. Proceedings of the National Acadamy of Sciences of USA 1973;70:3590-.

[15] Wheldhon TE, Kirk J, Grey WW. Mitotic autoregulation, growth control and neoplasia. Journal of Theoretical Biology 1973;38:627-639. 